\title{
Interobserver variability and likelihood of malignancy for fifth edition BI-RADS MRI descriptors in non-mass breast lesions
}

\author{
Magdalena Lunkiewicz ${ }^{1}$ - Serafino Forte ${ }^{1} \cdot$ Bianka Freiwald $^{2} \cdot$ Gad Singer $^{3} \cdot$ Cornelia Leo $^{4} \cdot$ Rahel A. Kubik-Huch $^{1}$ (D)
}

Received: 23 March 2019 / Revised: 20 May 2019 / Accepted: 7 June 2019/Published online: 7 August 2019

(C) The Author(s) 2019

\begin{abstract}
Objective Non-mass enhancement (NME) in breast MRI is the most common feature of ductal carcinoma in situ (DCIS). We sought to evaluate the interobserver variability and positive predictive value (PPV) for malignancy of NME descriptors using the fifth edition BI-RADS lexicon focusing on the newly introduced "clustered ring enhancement" pattern.

Materials and methods Breast MRIs of 129 patients who had undergone MRI-guided vacuum-assisted biopsy (VAB) in our institution were reviewed. Studies assessed as NME were classified according to the fifth edition BI-RADS lexicon by two breast radiologists. Consensus was reached by involving a third radiologist. Interobserver variability and PPV for malignancy were assessed. Results Seventy-two of 129 studies were assessed as NME. The disagreement rate in the first assessment step (mass vs. NME) was low at $9.3 \%(\kappa=0.81,95 \%$ confidence interval [CI] 0.71-0.91). The disagreement rate for distribution patterns was $23.6 \%$ $(k=0.67,95 \%$ CI $0.54-0.80)$ and $22.2 \%(k=0.69,95 \%$ CI $0.56-0.81)$ for internal enhancement patterns. Clustered ring enhancement (PPV 53.85, $p=0.038$ ) and segmental distribution (PPV 62.5\%, $p=0.028$ ) had the highest malignancy rates among internal enhancement and distribution patterns with a significant result; the combination of clustered ring enhancement and segmental distribution raised the malignancy rate by approximately 4\% (PPV 66.67\%, $p=0.049$ ).

Conclusion There was a high agreement rate among readers when differentiating NME from mass lesions. The agreement rate was lower when assessing the distribution and internal enhancement pattern descriptors, but still substantial. The descriptors clustered ring enhancement and segmental distribution were significant predictors of malignancy.

Key Points

- Non-mass enhancement is a common morphological feature of non-invasive breast cancer (DCIS) in MRI. Differentiation between potentially malignant and benign changes may be very challenging.

- Since clustered ring enhancement and segmental distribution are both significant predictors of malignancy, the awareness of this important finding, combined with high-quality image interpretation skills, may improve the tumor detection rate.

- The combination of clustered ring enhancement and segmental distribution increases the positive predictive value for malignancy, which may be relevant for clinical practice.
\end{abstract}

Keywords Image-guided biopsy $\cdot$ DCIS $\cdot$ Breast $\cdot$ Magnetic resonance imaging $\cdot$ Interobserver variability

Magdalena Lunkiewicz

Magdalena.Lunkiewicz@ksb.ch

1 Department of Radiology, Kantonsspital Baden, Im Ergel 1, CH-5404 Baden, Switzerland

2 Medizinisch Radiologisches Institut (MRI) Bahnhofplatz, Bahnhofplatz 3, 8001 Zürich, Switzerland

3 Department of Pathology, Kantonsspital Baden, Im Ergel 1, CH-5404 Baden, Switzerland

4 Department of Gynaecology, Kantonsspital Baden, Im Ergel 1, CH-5404 Baden, Switzerland

$\begin{array}{ll}\text { Abbreviations } \\ \text { ACR } & \text { American College of Radiology } \\ \text { B3 } & \text { Lesions of unknown biological potential } \\ \text { BPE } & \text { Background parenchymal enhancement } \\ \text { DCIS } & \text { Ductal carcinoma in situ } \\ \text { FOV } & \text { Field of view } \\ \text { MIBB } & \text { Minimally Invasive Breast Biopsies Working Group } \\ \text { NME } & \text { Non-mass enhancement } \\ \text { OR } & \text { Odds ratio } \\ \text { PACS } & \text { Picture archiving and communication system } \\ \text { TE } & \text { Echo time }\end{array}$


TR Repetition time

VAB Vacuum-assisted breast biopsy

\section{Introduction}

Magnetic resonance imaging (MRI) has the highest sensitivity (88-100\%) among breast imaging modalities [1-3]. Its relatively lower specificity of $72 \%$ [1] is considered to be its main disadvantage [4].

In the literature, MRI findings are reported to correlate with second-look ultrasonography examinations in 11-65\% [5-7] of cases; this wide range might be attributable to factors such as equipment, experience of the radiologists, patient selection, or type of the lesion (mass, NME, or focus). Lesions that are only detectable on MRI ("MRI-only lesions") attest to its high sensitivity and to the crucial role of MRI in breast diagnostic [8], especially in high-risk patients.

According to the Breast Imaging Reporting and Data System Lexicon (BI-RADS) [9], non-mass enhancement (NME) represents an area of contrast enhancement without a space-occupying effect. NME is the most common morphologic feature of ductal carcinoma in situ (DCIS). It does not have a sonographic correlate in the majority of cases (NME correlation rate $12 \%$ vs. mass lesion correlation rate $65 \%$ ) [7, 10], which implies the need for MRI-guided biopsy. Evaluation of NME lesions is challenging and it is associated with more false-positive results in comparison with enhancing mass lesions [11-14]. High-quality imaging-based characterization, while subject to interobserver variability, may improve the differentiation between potentially malignant and benign lesions and possibly contribute to the reduction of unnecessary biopsies.

BI-RADS lexicon is the main source of breast imaging terminology, reporting standards, and classification systems for mammography, ultrasound, and MRI of the breast. It provides a uniform assessment structure with recommendations for management. This facilitates intra- and interdisciplinary communication. The first edition of the BI-RADS lexicon was published 1993, followed by four more editions in 1995, 1998, 2003, and the currently used fifth edition in 2013. In the fifth edition of the BI-RADS lexicon [9], a few MRI terms for internal enhancement and distribution patterns of NME have been slightly changed. In the category of distribution patterns, "ductal enhancement" has been eliminated [9, 15]. Also the internal enhancement pattern descriptor "reticular/dendritic" has been omitted, while the descriptor "clustered ring enhancement" has been introduced. Clustered ring enhancement pattern is defined as multiple thin rings of enhancement, made visible around the ducts due to the enhancement of periductal stroma [14]. According to several studies [16-21], clustered ring enhancement is associated with one of the highest malignancy rates (Fig. 3).
The goal of our study was to assess interobserver variability when evaluating breast lesions on MRI according to the fifth edition of the BI-RADS MRI lexicon, as well as to evaluate the positive predictive value (PPV) for malignancy of each NME subtype, focusing on the newly introduced descriptor "clustered ring enhancement." Awareness of the values in malignancy prediction for NME descriptors, combined with high-quality image interpretation skills, may improve the tumor detection rate.

\section{Materials and methods}

\section{Patients}

This study was approved by the hospital ethics committee (project ID: 2017-00333). Because of the retrospective nature of the data retrieval, specific written consent was waived.

All patients $(n=144)$ who had undergone an MRI-guided vacuum-assisted core breast biopsy (VAB) between January 2011 and May 2017 in our institution were identified from the Picture Archiving and Communications System (PACS).

The inclusion criterion was the presence of the histopathological diagnosis based on the MRI-guided biopsy.

\section{Breast MRI examination, MRI-guided VAB, and histopathological diagnosis}

Diagnostic MRI examinations in our institution were performed in the prone position, using a 1.5-T scanner (Magnetom Aera ${ }^{\circledR}$, Siemens Healthcare) in 89 cases and a 3-T scanner (Skyra ${ }^{\circledR}$, Siemens) in 11 cases, as it had just become available at our new site. All examinations have been performed with a dedicated breast coil (18 channels). From the total number of 144 initially identified MRI examinations prompting an MRI-guided biopsy, 34 cases have been performed in external institutions (Siemens Avanto ${ }^{\circledR}$, Area ${ }^{\circledR}$, Espree ${ }^{\circledR}$, Verio ${ }^{\circledR}$; Philips Healthcare Ingenia ${ }^{\circledR}$, Philips Inc.). Of the 34 external MRI examinations, 19 cases were performed on a 1.5-T scanner, 10 cases were performed on a 3$\mathrm{T}$ scanner, and 5 examinations were excluded because of incomplete data sets, resulting in a total of 129 data sets for analysis. This resulted in a total number of 108 cases performed on a 1.5-T and 21 cases performed on a 3-T scanner.

The imaging protocol for the 1.5-T system included precontrast turbo spin-echo, T2-weighted axial images (T2WI) (repetition time TR $5600 \mathrm{~ms}$; echo time TE $110 \mathrm{~ms}$; FOV $360 \mathrm{~mm}$; matrix 512; section thickness $3.0 \mathrm{~mm}$ ), one precontrast dynamic T1-weighted axial (T1WI), and five postcontrast dynamic fat-saturated axial T1WI (repetition time TR $4.87 \mathrm{~ms}$; echo time TE $2.39 \mathrm{~ms}$; FOV $360 \mathrm{~mm}$; matrix 448; section thickness $1.0 \mathrm{~mm}$, flip angle $10^{\circ}$ ). The postcontrast series images were acquired $1 \min 45 \mathrm{~s}, 3 \min 14 \mathrm{~s}$, 
$4 \mathrm{~min} 44 \mathrm{~s}, 6 \mathrm{~min} 14 \mathrm{~s}$, and $8 \mathrm{~min} 30 \mathrm{~s}$ after the intravenous injection of Gd-DOTA (Dotarem $®$, Guerbet) $(0.1 \mathrm{~mL} / \mathrm{kg}$ body weight).

In the post-processing step, image subtractions were performed for each phase in the axial plane. In the sagittal and coronal planes, the subtractions were obtained in the early phase. Motion artifacts were corrected using commercial software (3 D Elastic Motion Correction $®$, Siemens Medical Solutions).

The MRI-guided VAB technique, as previously described by our group [8, 22], was performed according to the Swiss Minimally Invasive Breast Biopsies Working Group (MIBB) guidelines [23], with an 8-gauge needle [24].

\section{Image interpretation}

Two experienced breast radiologists (R.K. and S.F., 20 and 10 years of experience, respectively) independently evaluated all cases de novo using the fifth edition BI-RADS MRI lexicon. Both readers were unaware of the patients' clinical details, initial radiological reports, and the pathological results. In case of discrepancy, the findings were reviewed together with a third radiologist to achieve consensus. According to the
ACR MRI lexicon, lesions were first classified as NME [9] or mass lesions (Fig. 1). These findings were visually assessed, based on the morphologic characteristics in the turbo spinecho T2WI sequence in the axial plane (presence/absence of mass effect) and post-contrast dynamic T1WI sequences (presence/absence of interspersed normal parenchyma or fat tissue in the enhancing area). All MRI examinations assessed as NME were included for further analysis and subsequently evaluated in the post-contrast dynamic (delay, $1.45 \mathrm{~min}$ ) and subtraction images (axial, coronal, and sagittal planes) according to the enhancement distribution modifiers (Figs. 1 and 2) and the pattern of internal enhancement (Figs. 1 and 3).

In case of a clustered ring enhancement pattern, the absence of micro-cystic changes, which would indicate benignity, was confirmed in a T2-weighted sequence.

The BI-RADS classification from the original report had been recorded, since both readers were biased knowing that a biopsy had been performed.

\section{Statistical analysis}

With histopathological diagnosis as the reference standard, PPVs of the internal enhancement and global distribution

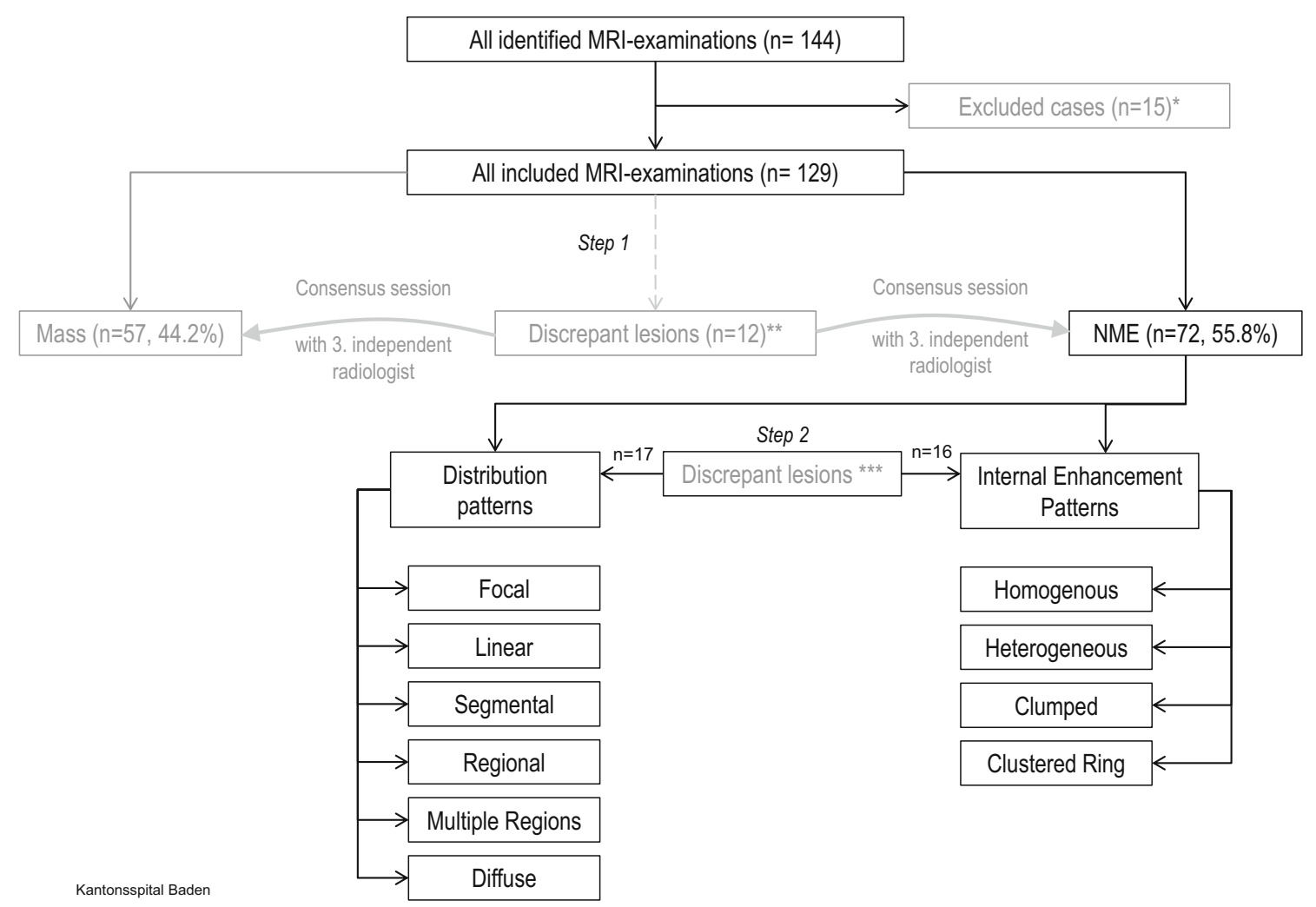

Fig. 1 Flowchart of the overall design, which involves the key steps of the prospective lesion evaluation, according to the revised BI-RADS lexicon (fifth edition). Step 1: General assessment (mass or NME). Step 2: Evaluation of the internal enhancement and distribution patterns.

*Incomplete data set; **12/129, disagreement rate 9.3\%, kappa 0.81 (CI $0.71-0.91) ; * * *$ Discrepant cases in distribution pattern $17 / 72$, disagreement rate $23.6 \%$, kappa 0.67 (CI 0.54-0.80). Discrepant cases in internal enhancement pattern $16 / 72$, disagreement rate $22.2 \%$, kappa 0.68 (CI 0.55-0.81) 

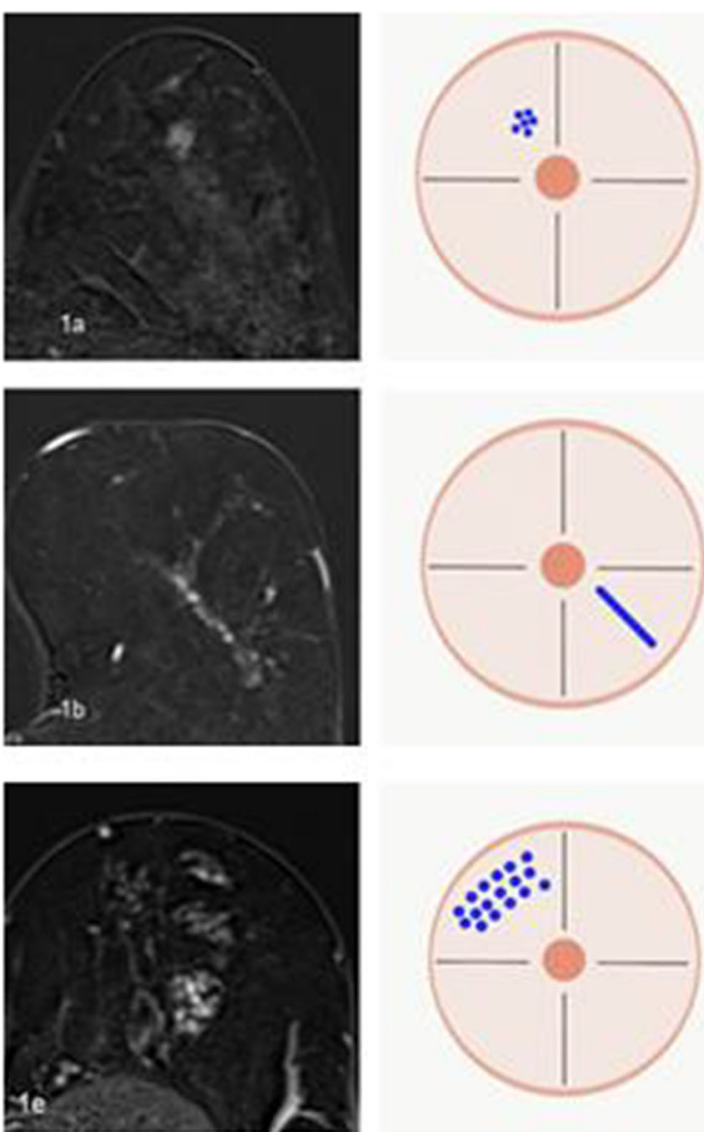

Fig. 2 Example cases from our daily practice (Department of Radiology, Kantonsspital Baden, Switzerland) for enhancement distribution patterns according to the fifth edition of BI-RADS lexicon [9]. 1a Focal $(<25 \%$ of quadrant, fat or normal glandular tissue interspersed among the enhancing components): A 49-year-old woman with usual ductal hyperplasia and fibrosis. 1b Linear (enhancement in a line, may be branching): A 75-yearold woman with linear clumped enhancement pattern and proven ductal carcinoma in situ. 1c Segmental (triangular, apex pointing to nipple,

patterns for determining malignancy were calculated as the proportion of malignant lesions to the corresponding pattern.

Any histopathological result other than DCIS or invasive carcinoma was considered benign; B3 lesions ("lesions of unknown biological potential") [25], which had not undergone subsequent surgery, were excluded from our statistical analysis.

PPV was calculated for each descriptor of internal enhancement and distribution patterns and for their different combinations.

To compare the PPVs of different enhancement patterns, odds ratios (OR) and Fisher's exact tests were calculated as follows: PPV of the corresponding pattern (malignant/nonmalignant) vs. PPV of the other patterns.

To evaluate interobserver agreement, the number of discrepant assessments (reader 1 vs. reader 2) was determined and Cohen's kappa (к) was calculated. The agreement rate was calculated for both steps (NME vs. mass lesion followed by evaluation of both distribution and internal enhancement
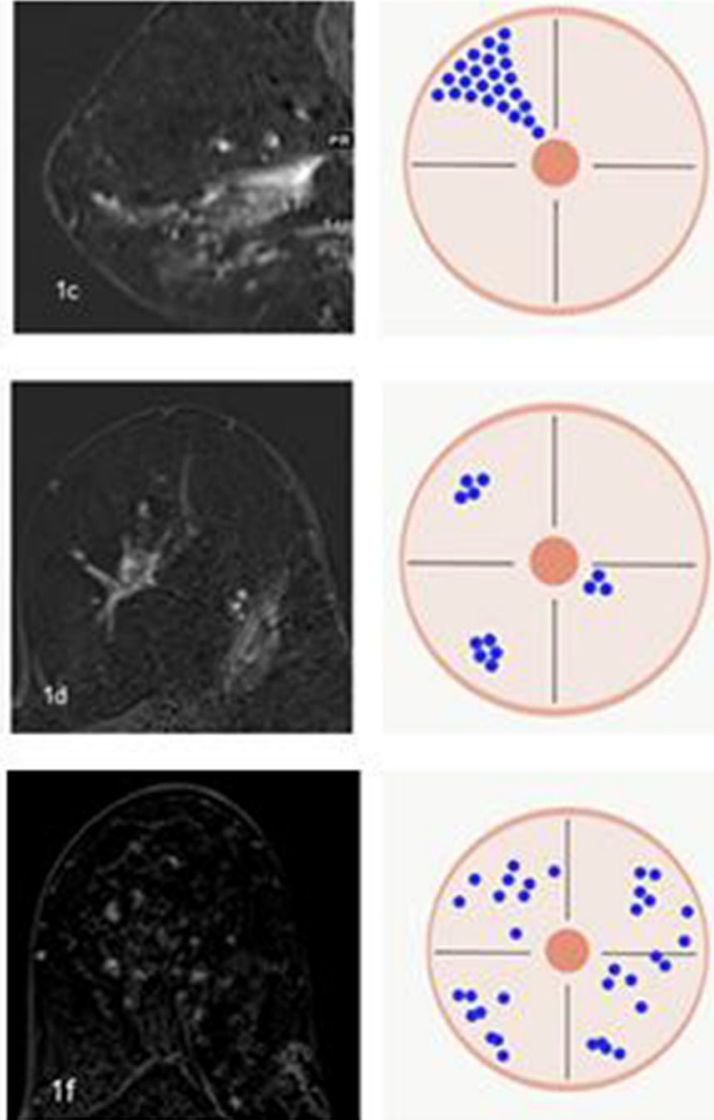

suggests ductal enhancement): A 60-year-old woman with invasive lobular carcinoma. 1d Multiple regions (enhancement over at least two large volumes of tissue): A 74-year-old woman with infiltrations of invasive lobular carcinoma. 1e Regional (encompasses more than a single duct system): A 52-year-old woman with normal parenchyma (dense breasts). 1f Diffuse (distributed randomly throughout the breast): A 47-year-old woman with fibrocystic changes

patterns) of prospective lesion evaluation (Fig. 1) as well as separately for internal enhancement and distribution pattern assessment. Cohen's kappa values were measured according to the method of Landis and Koch [26].

Descriptive statistics are presented as counts and frequencies for categorical data and mean (standard deviation) for metric variables.

A $p$ value $<0.05$ was considered significant.

Statistical analysis was performed by using open-source statistical software (R version 3.1.1) [27].

\section{Results}

\section{Demographics of the study population}

The patients' ages in the study population ranged from 31 to 91 with a mean age of 57.1 years $( \pm 11.6)$. 

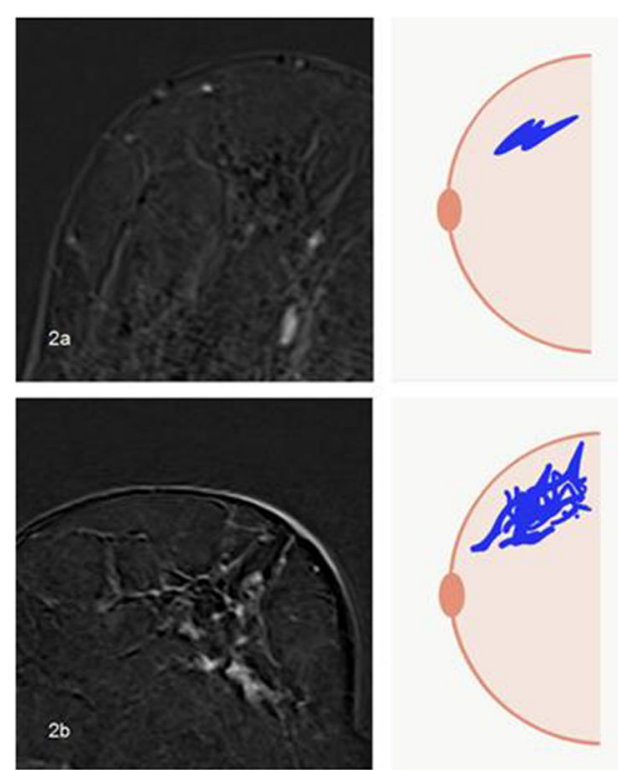

Fig. 3 Example cases from our daily practice (Department of Radiology, Kantonsspital Baden, Switzerland) for internal enhancement patterns according to the 5th edition of BI-RADS lexicon [9]. 2a Homogeneous: A 55-year-old woman with usual ductal hyperplasia. 2b Heterogeneous:

From the total number of included studies $(n=129), 72 /$ $129(55.8 \%)$ were classified as NME. The patients' ages in the NME group ranged from 31.5-91 with a mean age of 52.6 years $( \pm 20.8)$.

Of the 72 cases, $18 / 72(25 \%)$ had a positive family history of breast cancer (as declared by the patient on the MIBBquestionnaire; lifetime risk was not recorded), 17/72 (24\%) had no family history, and 37/72 (51\%) were not specified.

The study group identified to access the PPV for malignancy (after exclusion of the $\mathrm{B} 3$ lesions, which had not undergone surgery) included the total number of 67 cases. The patients' age in this group ranged from 31.5-81 with a mean age of 51.5 years $( \pm 20.7)$.

Of the 67 cases, 17/67 (25.4\%) had a positive family history of breast cancer (as declared by the patient on the MIBBquestionnaire; lifetime risk was not recorded), 15/67 (22.4\%) had no family history, and 35/67 (52.2\%) were not specified.

In the overall study population group, $60 \%$ (78/129) of lesions had been classified as BI-RADS 4, 24\% (31/129) as BI-RADS 3, 3\% (4/129) as BI-RADS 5, and 13\% (16/129) had unrecorded BI-RADS classifications (mostly images from external sites).

\section{Interobserver agreement}

The interobserver agreement rates for each of the assessment steps are shown in Figs. 1 and 4.

The interobserver agreement rate in the second assessment step (evaluation of internal enhancement and distribution patterns) was lower than in the first assessment step (NME vs.
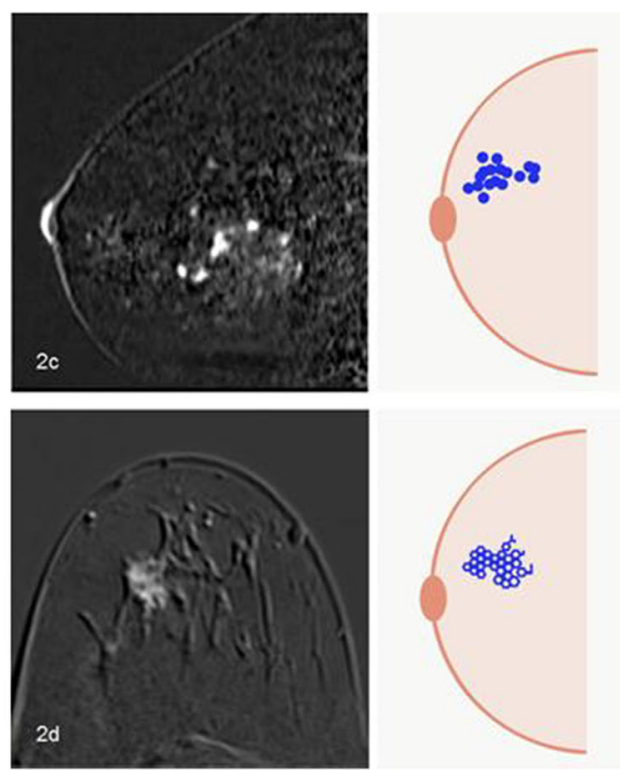

A 56-year-old woman with apocrine focal metaplasia. 2c Clumped: A $67-$ year-old woman with pseudoangiomatous stromal hyperplasia and fibrosis. 2d Clustered ring enhancement: A 65-year-old woman with invasive lobular carcinoma

mass lesion), but still substantial for evaluation of both distribution and internal enhancement patterns (Fig. 4).

\section{Overlap of clustered ring enhancement and other internal enhancement patterns in the visual assessment process (interobserver agreement on clustered ring enhancement)}

In 18 cases, one or both readers assessed a study as clustered ring enhancement. In 8/18 cases, the visual assessment between both readers was discrepant (44\% disagreement rate). The total number of cases with a definite assessment as clustered ring enhancement after the consensus session with a third independent radiologist was $n=14$. In $10 / 14$ studies, the visual assessment between both readers was concordant ( $71 \%$ agreement rate).

Of the four remaining discrepant studies, 3 studies were assessed in consensus as a clumped enhancement, and the other 3 were assessed as heterogeneous. There were no further intersections between clustered ring enhancement and other internal enhancement patterns (Table 1).

\section{Distribution of internal enhancement and distribution patterns in the NME group}

In the overall NME group, the most frequent distribution pattern was focal $(n=28 / 72,38.9 \%)$, followed by linear $(n=20 /$ $72,27.8 \%)$, regional $(n=13 / 72,18.1 \%)$, segmental $(n=9 / 72$, $12.5 \%$ ), and multiple regions ( $n=2 / 72,2.78 \%)$ (Fig. 5a). 
Step 1

NME vs. Mass

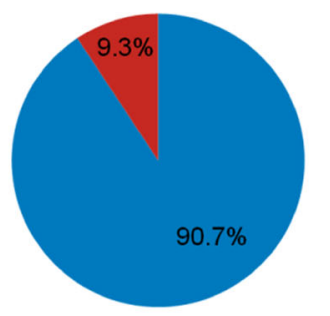

Cohen`s Kappa $0.81^{*}, \mathrm{Cl}(0.71-0.91)$

Step 2

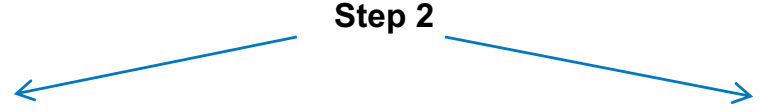

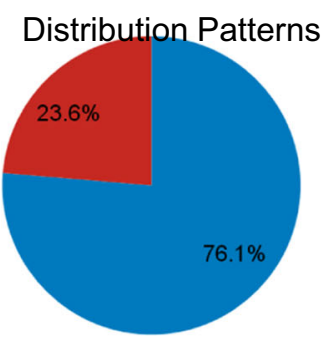

Cohen`s Kappa: $0.67^{* *}, \mathrm{Cl}$ 0.54-0.80

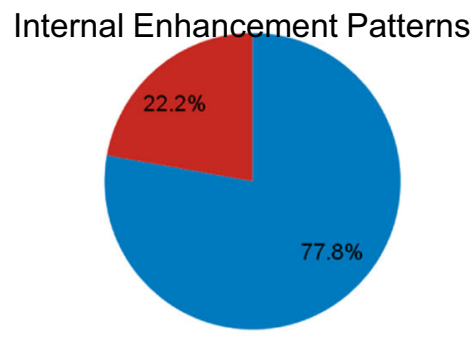

Cohen`s Kappa: $0.69^{* *}, \mathrm{Cl}$ 0.56-0.81).

Fig. 4 Interreader agreement. * $0-0.20$, slight agreement; $0.21-0.40$, moderate agreement; $0.61-0.80$, substantial agreement; and $0.81-1$, almost perfect agreement

The most frequent internal enhancement pattern was clumped $(n=30 / 72,41.7 \%)$, followed by heterogeneous ( $n=17 / 72,23.6 \%)$, clustered ring $(n=14 / 72,19.4 \%)$, and homogenous ( $n=11 / 72,15.3 \%$ ) (Fig. $5 b)$.

The distribution of internal enhancement and distribution patterns in the study group identified in particular to access the PPV for malignancy correspond to the described above distribution in the overall NME group. The most frequent distribution pattern was focal $(n=28 / 67,41.8 \%)$, followed by linear $(n=18 / 67,26.9 \%)$, regional $(n=11 / 67,16.4 \%)$, segmental $(n=8 / 67,11.9 \%)$, and multiple regions $(n=2 / 67,3 \%)$.

The most frequent internal enhancement pattern was clumped $(n=27 / 67,40.3 \%)$, followed by heterogeneous $(n=17 / 67,25.4 \%)$, clustered ring $(n=13 / 67,19.4 \%)$, and homogenous $(n=10 / 67,14.9 \%)$.

\section{Histopathological findings in the NME group}

The frequency of malignant findings in the overall NME group in our study population was $24.6 \%$ (19/72 cases).

Of the $53(73.6 \%)$ benign findings, 13 lesions were B3 lesions ( $18.1 \%$ of the total study population). Eight patients with a B3 lesion had subsequently undergone surgical resection and no upstaging had been reported (Fig. 6). The five remaining B3 lesions, which had not undergone subsequent surgery, were excluded from our statistical analysis accessing the PPV for malignancy, due to the unknown upstage rate. The frequency of malignant findings in the consecutive study group was $28.4 \%$ (19/67 cases). The distribution of both malignant and benign histopathological diagnoses in both study groups (before and after the exclusion of the selected B3 lesions) is shown in Table 2.
Table 1 Intersections between clustered ring enhancement and other internal enhancement patterns

\begin{tabular}{llllll}
\hline & \multicolumn{2}{c}{ Reader 1 } & & & \\
\cline { 2 - 6 } Reader 2 & Clumped & Clustered ring & Heterogeneous & Homogeneous & Rim enhancement (mass) \\
\hline Clumped & & 3 & & & \\
Clustered ring & 1 & 10 & 0 & 0 & \\
Heterogeneous & 2 & & & \\
Homogeneous & 0 & & & \\
Rim enhancement (mass) & 1 & & & \\
\hline
\end{tabular}


Fig. 5 a, b Distribution of distribution pattern and internal enhancement pattern descriptors in the study population

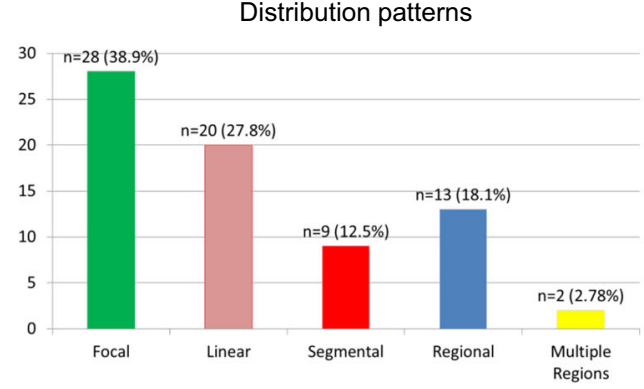

a
Internal enhancement patterns

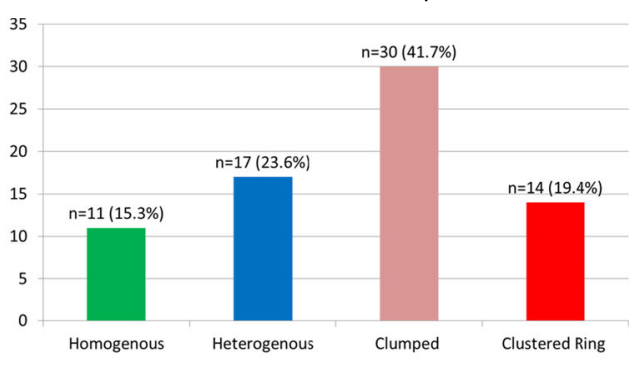

b

\section{Positive predictive value for malignancy}

Of the internal enhancement patterns, clustered ring enhancement had the highest positive predictive value (PPV) for malignancy, significantly higher in comparison with other internal enhancement patterns $(53.85 \%, 7$ malignant lesions of a total number of 13 cases, $p=0.038$ ). Clumped enhancement, heterogeneous enhancement, and homogenous enhancement were not significant predictors of malignancy (Table 3).

Among the distribution patterns, segmental distribution had the highest PPV for malignancy, which was significantly higher in comparison with other distribution patterns $(62.5 \%$ $\%, 5$ malignant lesions of a total number of 8 cases, $p=0.028$ ). Linear, focal, and regional enhancements were not significant predictors.

The combination of clustered ring enhancement and segmental distribution increased the PPV by approximately $4 \%$ to
$66.67 \%$ (4 malignant lesions of a total number of $6, p=0.049$ ) and represented the highest PPV for malignancy among all possible combinations of internal enhancement and distribution patterns, with a marginally significant result.

\section{Discussion}

As assessment of NME is very challenging, being aware of the interobserver variabilities and their characteristics may improve the quality of reporting. To our knowledge, our study is the first one assessing the interobserver agreement after the publication of the fifth edition BI-RADS lexicon. The interobserver agreement of our study varied strongly between the first assessment step (NME vs. mass lesions) and the second assessment step (differentiation of NME descriptors). The interobserver agreement of the first named assessment step was almost perfect; however, the experience of both readers (20 and 10 years of

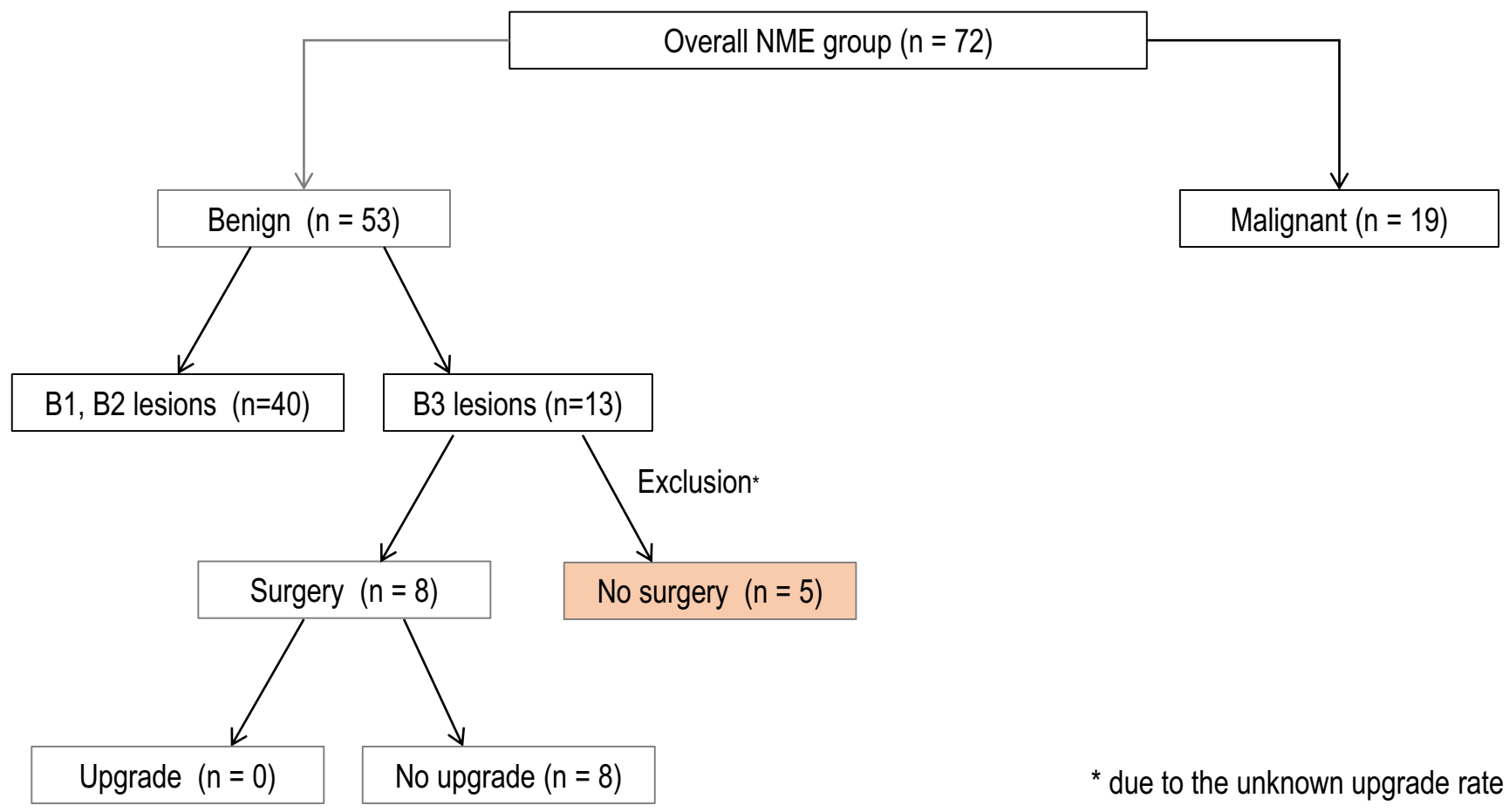

Fig. 6 Histopathological diagnoses in the NME group 
Table 2 Distribution of histopathological diagnosis in the overall NME group and NME group*

\begin{tabular}{|c|c|c|c|c|c|c|c|}
\hline \multicolumn{4}{|c|}{ Overall NME group $(n=72)$} & \multicolumn{4}{|l|}{ NME group* $(n=67)$} \\
\hline Benign & $53(73.6 \%)$ & Malignant & $19(26.4 \%)$ & Benign & $48(71.6 \%)$ & Malignant & $19(28.4 \%)$ \\
\hline Fibrosis & $10(13.8 \%)$ & DCIS & $6(8.3 \%)$ & Fibrosis & $10(14.9 \%)$ & DCIS & $6(9 \%)$ \\
\hline UDH & $6(8.3 \%)$ & IDC & $5(6.9 \%)$ & UDH & $6(9 \%)$ & IDC & $5(7.5)$ \\
\hline $\mathrm{ADH}$ & $5(6.9 \%)$ & ILC & $3(4.1 \%)$ & $\mathrm{ADH}$ & $3(4.5 \%)$ & ILC & $3(4.5 \%)$ \\
\hline Papilloma $\varnothing$ atypia & $5(6.9 \%)$ & IDC with DCIS & $1(1.7)$ & Papilloma $\varnothing$ atypia & $4(6 \%)$ & IDC with DCIS & $1(1.5 \%)$ \\
\hline Periductal mastitis & $5(6.9 \%)$ & Other malignancy & $4(5.5 \%)$ & Periductal mastitis & $5(7.5 \%)$ & Periductal mastitis & $4(6 \%)$ \\
\hline Fibroadenoma & $4(5.5 \%)$ & & & Fibroadenoma & $4(6 \%)$ & & \\
\hline Sclerosing adenosis & $4(5.5 \%)$ & & & Sclerosing adenosis & $4(6 \%)$ & & \\
\hline FEA & $2(2.7 \%)$ & & & FEA & $2(3 \%)$ & & \\
\hline Papilloma with atypia & $2(2.7 \%)$ & & & Papilloma with atypia & $1(1.5 \%)$ & & \\
\hline Normal parenchyma & $1(1.7 \%)$ & & & Normal parenchyma & $1(1.5 \%)$ & & \\
\hline Other benign results & $9(12.5)$ & & & Other benign results & $8(11.9 \%)$ & & \\
\hline
\end{tabular}

$U D H$ usual ductal hyperplasia, $A D H$ atypical ductal hyperplasia, FEA flat epithelial atypia, DCIS ductal carcinoma in situ, IDC invasive ductal carcinoma, ILC invasive lobular carcinoma

*Consecutive study group after exclusion of the B3 lesions without surgery

experience in breast imaging) should be taken into account. The interobserver agreement in the assessment of NME internal enhancement and distribution patterns was considerably lower (by around 14\%). Especially in the evaluation of the distribution and internal enhancement patterns, a second reader opinion may be useful. There were overlaps between clustered ring, clumped, and heterogeneous internal enhancement patterns, whose assessment should be perceived as very challenging, especially when performed by less experienced readers.

Apart from the interobserver variability, the results showed that clustered ring enhancement and segmental distribution pattern are significantly more frequently associated with malignancy in comparison with other NME descriptors. This finding is concordant with several previous studies [17, 21, 28, 29]. There are prior studies both with higher and lower absolute results, but

Table 3 Positive predictive value for malignancy of distribution patterns and internal enhancement patterns

\begin{tabular}{llllll}
\hline & Total $(n)$ & Malignant & PPV & $p$ value & OR \\
\hline Clustered ring & 13 & 7 & 53.85 & $0.038^{*}$ & 3.982 \\
Clumped & 27 & 7 & 25.93 & 0.788 & 0.819 \\
Heterogeneous & 17 & 3 & 17.65 & 0.356 & 0.460 \\
Homogeneous & 10 & 2 & 20 & 0.712 & 0.593 \\
Focal & 28 & 5 & 17.86 & 0.177 & 0.440 \\
Linear & 18 & 5 & 27.78 & 1 & 1.064 \\
Segmental & 8 & 5 & 62.5 & $0.028^{*}$ & 5.702 \\
Regional & 11 & 2 & 18.18 & 0.714 & 0.559 \\
Multiple regions & 2 & 1 & 50 & 0.468 & 2.772 \\
\hline
\end{tabular}

Odds Ratios and $p$ values from corresponding Fisher's exact tests were calculated based on the comparison between the PPV of the corresponding pattern (malignant/non-malignant) and other patterns

$* p<0.05$ was considered significant there is only one prior study [17] performed after the publication of the revised fifth edition of BI-RADS lexicon (Table 4). This study, by Chikarmane et al, departs from our study in a few aspects. The PPV of clustered ring enhancement for malignancy in our study was $53.85 \%$, which was slightly higher than that observed by Chikarmane et al (44\%). This may be related to a different patient selection process; e.g., clustered ring enhancement studies with micro-cystic changes in T2WI sequences were excluded from biopsy, since the coexistence of clustered ring enhancement and micro-cystic changes may be associated with a benign result (mastopathy) [30]. This improved the specificity of our result. Another difference in our study, compared with that of Chikarmane et al, which used two readers, is the achievement of consensus via a third, independent radiologist.

Tozaki et al [21] and Uematsu et al [29] documented a significantly higher malignancy rate and PPV of clustered ring enhancement (96\% and 77\%, respectively) (Table 4). This may be associated with a different visual assessment concept in those studies, since they perceived the clustered ring internal enhancement descriptor mainly as a subtype/supplement to the heterogeneous and clumped enhancement pattern, as their studies were performed before the publication of the 5th edition of the BI-RADS lexicon 2014. The higher frequency rates of clustered ring enhancement, documented by those two prior studies (Tozaki et al, 23/61-38\%; Uematsu et al, 66/ 124-53\%; our study, 13/67-19.4\%) and the different methods of confirmation of the histopathological diagnosis between the trials (our study, MRI-guided VAB in $100 \%$ of cases; Tozaki et al, additional mastectomy and lumpectomy in $60 \%$ of cases) may have influenced the final results.

Among the distribution modifiers, segmental distribution pattern had a significantly higher PPV for malignancy with $62.5 \%$ $(p=0.028)$. This finding is consistent with previously published 
Table 4 Tabulated listing of the literature results on clustered ring enhancement and segmental distribution in comparison with our findings

\begin{tabular}{|c|c|c|c|c|c|}
\hline Study & $\begin{array}{l}\text { Total no. of } \\
\text { included NME } \\
\text { lesions }\end{array}$ & $\begin{array}{l}\text { No. of clustered ring } \\
\text { enhancement lesions/ } \\
\text { total }(\%)\end{array}$ & $\begin{array}{l}\text { No. of malignant cases/total } \\
\text { no. of clustered ring } \\
\text { enhancement lesions (PPV \%) }\end{array}$ & $\begin{array}{l}\text { No. of lesions } \\
\text { with segmental } \\
\text { distribution/total }(\%)\end{array}$ & $\begin{array}{l}\text { No. of malignant lesions/total } \\
\text { no. of lesions with segmental } \\
\text { distribution (PPV\%) }\end{array}$ \\
\hline Tozaki et al [21] & 61 & $23 / 61(37 \%)$ & $22 / 23(96 \%)$ & $19 / 61(31 \%)$ & $19 / 19(100 \%)$ \\
\hline Uematsu et al [29] & 124 & $66 / 124(53 \%)$ & $51 / 66(77 \%)$ & $53 / 124(43 \%)$ & $45 / 53(85 \%)$ \\
\hline Chikarmane et al [17]* & 144 & $30 / 144(21 \%)$ & $11 / 30(37 \%)$ & $25 / 144(17 \%)$ & $10 / 25(40 \%)$ \\
\hline Our study* & 67 & $13 / 67(19.4 \%)$ & $7 / 13(53.85 \%)$ & $8 / 67(11.9 \%)$ & $5 / 8(62.5 \%)$ \\
\hline
\end{tabular}

*Performed after the introduction of the revised BI-RADS lexicon 5th edition (2014)

results [17, 21, 28, 29] (Table 4). The observed subtle decrease of the PPV of segmental distribution in comparison with Tozaki et al and Uematsu et al may be again related to our institution's different indications for MRI-guided VAB (MRI-only lesions). This suggests less distinct lesions in our study group (no sonographic correlate) and correspondingly lower malignancy rates, compared with the studies of Tozaki et al and Uematsu et al (malignancy rate Tozaki et al, 35/61 [57\%]; Uematsu et al, 85/ 124 [69\%]; our study, 19/67 [28.4\%]).

Our study has several limitations. This was a retrospective study.

The highest PPV for malignancy $(66.67 \%)$ was assessed for the combination of clustered ring enhancement and segmental distribution. However, this result has almost missed the significance level with a $p$ value of 0.049 , due to the relatively low number of cases.

The family history of breast cancer was recorded as declared by the patient on the MIBB questionnaire; lifetime risk was not calculated.

Since the included lesions were MRI-only lesions, which tended to be less distinct and subject to higher interobserver variability, we did not evaluate the lesion size; thus, we could not stratify for size. The potential overlap between larger NME lesions and background parenchymal enhancement (BPE) should be also taken into account.

In conclusion, the awareness of higher interobserver variability in the assessment of the distribution and internal enhancement patterns of NME lesions, as well as the awareness of the most frequent intersections between descriptors, is relevant for high-quality reporting and implies the requirement of a second (and possibly a third) reader opinion.

The newly introduced clustered ring enhancement and segmental distribution pattern are significant predictors of malignancy among the NME descriptors. The awareness of this important finding combined with high-quality image interpretation skills may improve the tumor detection rate. The combination of both patterns increases the PPV for malignancy rate by an extra 4\% (PPV of the combination of both, $66.7 \%$ ), which may be relevant for clinical practice.

Acknowledgments We thank Mr. Andreas Schötzau (Eudox Institute for Biomathematics) for his help in the statistical analysis.
We thank Ms. Libby Cone, MD, MA, from Edanz Group Japan, for editing the drafts of this manuscript.

Funding This study has received funding by internal funding sources/ Kantonsspital Baden.

\section{Compliance with ethical standards}

Guarantor The scientific guarantor of this publication is Rahel A. Kubik-Huch, MD, MPH.

Conflict of interest The authors of this manuscript declare no relationships with any companies, whose products or services may be related to the subject matter of the article.

Statistics and biometry Mr. Andreas Schötzau (Eudox Institute for Biomathematics) kindly provided statistical advice for this manuscript.

Informed consent Written informed consent was waived by the Institutional Review Board.

Ethical approval Institutional Review Board approval was obtained.
Methodology
- retrospective
- diagnostic study
- performed at one institution

Open Access This article is distributed under the terms of the Creative Commons Attribution 4.0 International License (http:// creativecommons.org/licenses/by/4.0/), which permits unrestricted use, distribution, and reproduction in any medium, provided you give appropriate credit to the original author(s) and the source, provide a link to the Creative Commons license, and indicate if changes were made.

\section{References}

1. Esserman LJ, Joe BN (2016) Diagnostic evaluation of women with suspected breast cancer. UpToDate, Topic 808, Version 36.0

2. Berg WA, Gutierrez L, NessAiver MS et al (2004) Diagnostic accuracy of mammography, clinical examination, US, and MR imaging in preoperative assessment of breast cancer. Radiology 233: 830-849

3. Gweon HM, Cho N, Seo M, Chu AJ, Moon WK (2014) Computeraided evaluation as an adjunct to revised BI-RADS atlas: improvement in positive predictive value at screening breast MRI. Eur Radiol 24:1800-1807 
4. Kavanagh AM, Giles GG, Mitchell H, Cawson JN (2000) The sensitivity, specificity, and positive predictive value of screening mammography and symptomatic status. J Med Screen 7:105-110

5. LaTrenta LR, Menell JH, Morris EA, Abramson AF, Dershaw DD, Liberman L (2003) Breast lesions detected with MR imaging: utility and histopathologic importance of identification with US. Radiology 227:856-861

6. DeMartini WB, Eby PR, Peacock S, Lehman CD (2009) Utility of targeted sonography for breast lesions that were suspicious on MRI. AJR Am J Roentgenol 192:1128-1134

7. Abe H, Schmidt RA, Shah RN et al (2010) MR-directed ("secondlook") ultrasound examination for breast lesions detected initially on MRI: MR and sonographic findings. Am J Roentgenol 194:370 377

8. Spick C, Schernthaner M, Pinker K et al (2016) MR-guided vacuum-assisted breast biopsy of MRI-only lesions: a single center experience. Eur Radiol 26:3908-3916

9. Morris EACC, Lee $\mathrm{CH}$ et al (2013) ACR BI-RADS $®$ magnetic resonance imaging. In: ACR BI-RADS ${ }^{\circledR}$ atlas, breast imaging reporting and data system. American College of Radiology

10. Howell A, Anderson AS, Clarke RB et al (2014) Risk determination and prevention of breast cancer. Breast Cancer Res 16:446

11. Baltzer PA, Benndorf M, Dietzel M, Gajda M, Runnebaum IB, Kaiser WA (2010) False-positive findings at contrast-enhanced breast MRI: a BI-RADS descriptor study. AJR Am J Roentgenol 194:1658-1663

12. Gutierrez RL, DeMartini WB, Eby PR, Kurland BF, Peacock S, Lehman CD (2009) BI-RADS lesion characteristics predict likelihood of malignancy in breast MRI for masses but not for nonmasslike enhancement. AJR Am J Roentgenol 193:994-1000

13. Gity M, Ghazi Moghadam K, Jalali AH, Shakiba M (2014) Association of different MRI BIRADS descriptors with malignancy in non mass-like breast lesions. Iran Red Crescent Med J 16

14. Chadashvili T, Ghosh E, Fein-Zachary V et al (2014) Nonmass enhancement on breast MRI: review of patterns with radiologicpathologic correlation and discussion of management. AJR Am J Roentgenol 204:219-227

15. Rao AA, Feneis J, Lalonde C, Ojeda-Fournier H (2016) A pictorial review of changes in the BI-RADS fifth edition. Radiographics 36 : 623-639

16. Sakamoto N, Tozaki M, Higa K et al (2008) Categorization of nonmass-like breast lesions detected by MRI. Breast Cancer 15(3): 241-246

17. Chikarmane SA, Michaels AY, Giess CS (2017) Revisiting nonmass enhancement in breast MRI: analysis of outcomes and follow-up using the updated BI-RADS atlas. AJR Am J Roentgenol 209:1178-1184

18. Cho YH, Cho KR, Park EK et al (2016) Significance of additional non-mass enhancement in patients with breast cancer on preoperative $3 \mathrm{~T}$ dynamic contrast enhanced MRI of the breast. Iran J Radiol. https://doi.org/10.5812/iranjradiol.30909
19. Asada T, Yamada T, Kanemaki Y, Fujiwara K, Okamoto S, Nakajima Y (2017) Grading system to categorize breast MRI using BI-RADS 5th edition: a statistical study of non-mass enhancement descriptors in terms of probability of malignancy. Jpn J Radiol. https://doi.org/10.1007/s11604-017-0717-9

20. Tozaki M, Fukuda K (2006) High-spatial-resolution MRI of nonmasslike breast lesions: interpretation model based on BI-RADS MRI descriptors. AJR Am J Roentgenol 187:330-337

21. Tozaki M, Igarashi T, Fukuda K (2006) Breast MRI using the VIBE sequence: clustered ring enhancement in the differential diagnosis of lesions showing non-masslike enhancement. AJR Am J Roentgenol 187:313-321

22. Imschweiler T, Haueisen H, Kampmann G et al (2014) MRI-guided vacuum-assisted breast biopsy: comparison with stereotactically guided and ultrasound-guided techniques. Eur Radiol 24:128-135

23. Kurtz C, Kubik-Huch RA, Kampmann G, Rageth C, Öhlschlegel C (2014) Interdisziplinäre Konsensus-Guidelines für dei Durchführung von MRT-gestützten Vakkumbiopsien der Schweizer Gesellschaft für Senologie (SGS) - erarbeitet durch die MIBB Arbeitsgruppe. Senologie 11:1-10

24. Saladin C, Haueisen H, Kampmann G et al (2016) Lesions with unclear malignant potential (B3) after minimally invasive breast biopsy: evaluation of vacuum biopsies performed in Switzerland and recommended further management. Acta Radiol 57:815-821

25. Perry N, Broeders M, de Wolf C, Toernberg S, Holland R, von Karsa L (2006) European guidelines for quality assurance in breast screening and diagnosis. Office for Official Publications of the European Communities, Luxembourg, pp 221-256

26. Landis JR, Koch GG (1977) The measurement of observer agreement for categorical data. Biometrics 33:159-174

27. R Core Team (2015) A language and environment for statistical computing. R Foundation for Statistical Computing, Vienna, Austria

28. Yang QX, Ji X, Feng LL et al (2017) Significant MRI indicators of malignancy for breast non-mass enhancement. J Xray Sci Technol 25:1033-1044

29. Uematsu T, Kasami M (2012) High-spatial-resolution 3-T breast MRI of nonmasslike enhancement lesions: an analysis of their features as significant predictors of malignancy. AJR Am J Roentgenol 198:1223-1230

30. Milosevic ZC, Nadrljanski MM, Milovanovic ZM, Gusic NZ, Vucicevic SS, Radulovic OS (2017) Breast dynamic contrast enhanced MRI: fibrocystic changes presenting as a non-mass enhancement mimicking malignancy. Radiol Oncol 51:130-136

Publisher's note Springer Nature remains neutral with regard to jurisdictional claims in published maps and institutional affiliations. 